\title{
Acute ST elevation MI with anterior descending artery obstruction after blunt chest wall trauma
}

\author{
Preeti Jadhav $^{1,2 *}$, Maleeha Zahid ${ }^{1}$, Bing Yue ${ }^{1,2}$ Gayathri Kamalakkannan $^{1,2}$, Claude Simon ${ }^{1,2}$, Jonathan N Bella1,2 and Timothy Vittorio ${ }^{1,2}$ \\ ${ }^{1}$ BronxCare Health System, USA \\ ${ }^{2}$ Mount Sinai Morningside - Mount Sinai Hospital, USA
}

\begin{abstract}
We present a case of anterior wall MI in a patient who was physically assaulted by a group people prior to ER arrival. Initial presentation consistent with musculoskeletal pain but due to abnormal EKG and continued chest pain further work was done that revealed single vessel CAD.
\end{abstract}

\section{History of presentation}

Our patient is a 57 year old male who presented to the Emergency Department (ED) with complaints of back pain and chest pain that started after he was physically assaulted 2 days ago by a group of people outside grocery store. He stayed home for 2 days but body ache persisted, so he came to ED. Chest pain was described as sharp, nonexertional, radiating to left arm, worsened by arm movement and there were no relieving factors. He denied any shortness of breath, nausea, vomiting, headache, or abdominal pain after trauma. At baseline, he reported excellent exercise tolerance. In the ED, he was afebrile, heart rate was 76 beats per minute, blood pressure was 180/100 $\mathrm{mm} \mathrm{Hg}$ and oxygen saturation was $99 \%$ on room air. On physical examination, the patient was not in mild distress due to pain in the back and chest. Heart sounds were normal, no audible murmur or gallop and no jugular venous distension was noted.

He had tenderness to palpation over chest wall and upper back. Lungs were clear to auscultation and abdomen was soft without any evidence of organomegaly. Patient had left 5th digit swelling and lefthand bruises otherwise no limb swelling was noted.

\section{Medical history}

The patient had well-controlled arterial hypertension and was an active smoker with 20 pack- years.

\section{Differential diagnosis}

Based on the information above, the differential diagnosis included musculoskeletal chest pain due to blunt trauma, cardiac contusion, stress induced cardiomyopathy or coronary heart disease.

\section{Investigations}

Initial laboratory examination was significant for the elevated high sensitivity troponin level at 3700ng/L, ProBNP 1523pg/ml and creatine kinase at 3662 unit/L. Otherwise no renal impairment, normal electrolytes and unremarkable cell counts. The patient's EKG showed normal sinus rhythm with ST elevation and T wave inversion in V2 to V4 leads along with Q waves in V2 to V4 (Figure 1). An urgent chest and abdominal angiogram showed no evidence of aortic

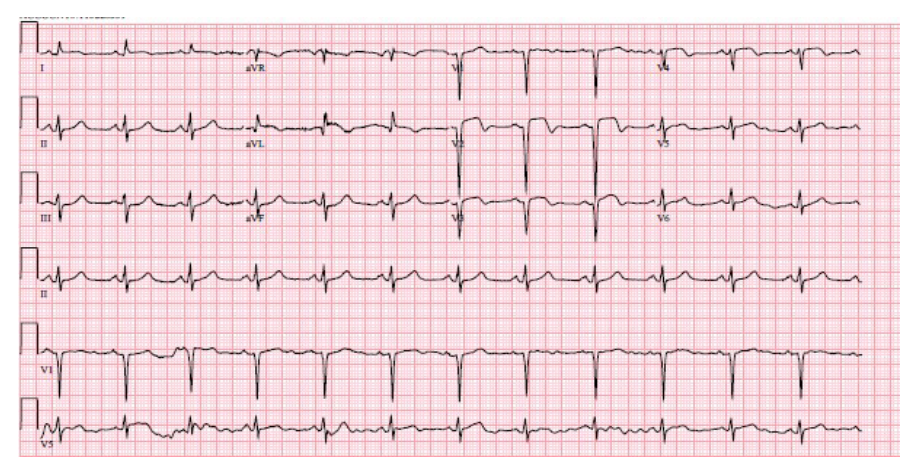

Figure 1. A 12-lead ECG shows ST segment elevations in V2-V4 along with Q waves in those leads

dissection or another intra-abdominal organ injury. Echocardiogram showed moderately reduced ejection fraction to $38 \%$ with grade 2 diastolic dysfunction. There were regional wall motion abnormalities with hypokinesis of the basal anteroseptal and the basal inferoseptal segments, akinesis of the mid to apical anterior and septal walls, apical inferior and lateral walls, and hyperkinesis of basal lateral, inferior and inferolateral walls. The study also showed moderate size fixed thrombus attached to the left ventricular (LV) apex.

\section{Management}

Patient was taken for emergent cardiac catheterization that showed $100 \%$ stenosis in proximal left anterior descending artery with TIMI 0 flow (Figures 2 and 3). There were abundant collaterals from RCA to septum (Figure 4). Due to late presentation MI with Q waves on EKG, PCI was deferred. Patient was started on aspirin, clopidogrel, carvedilol,

*Correspondence to: Preeti Jadhav, BronxCare Health System, Mount Sinai Morningside - Mount Sinai Hospital, 215 East 95th Street, New York, NY, 10128, USA, Tel: 646-460-7769, E-mail: Preeti.Jadhav@mountsinai.org

Received: October 06, 2020; Accepted: October 16, 2020; Published: October 21, 2020 


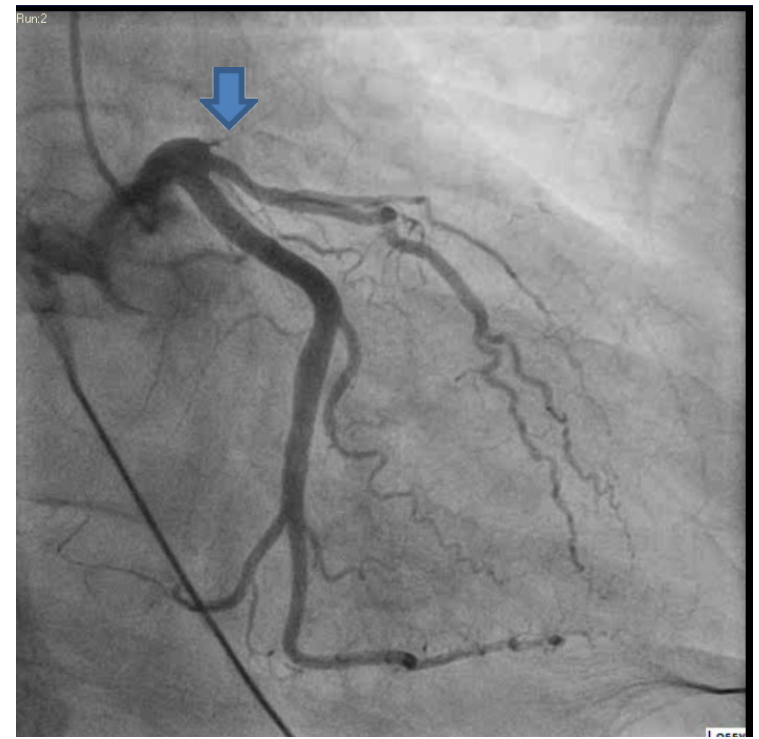

Figure 2. Coronary Angiogram in Right Anterior Oblique Caudal (RAO) view with complete occlusion of LAD as indicated by arrow

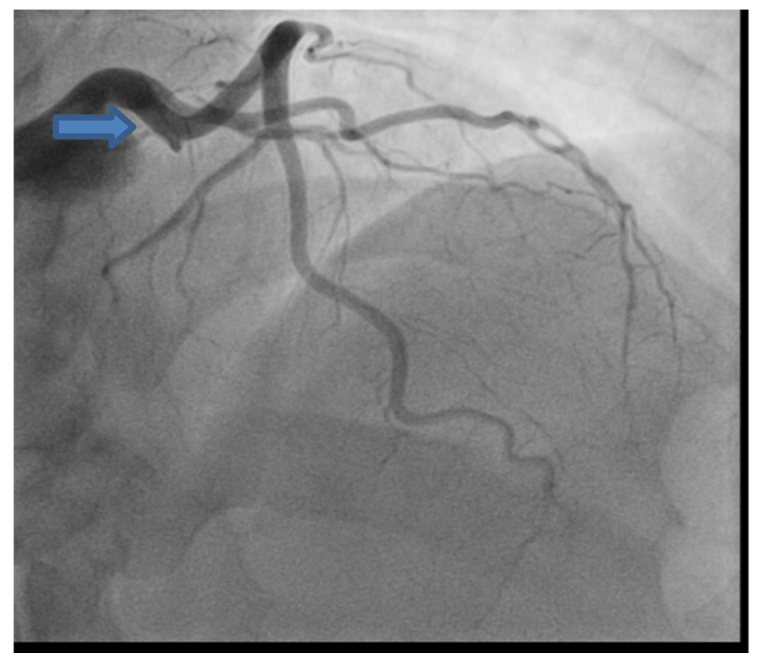

Figure 3. Coronary Angiogram in Right Anterior Oblique (RAO) Cranial view with complete occlusion of LAD as indicated by arrow

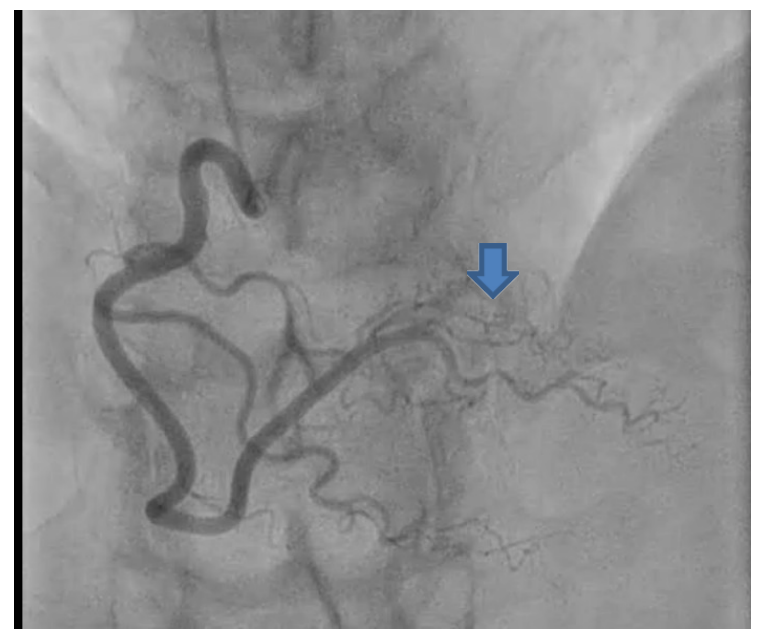

Figure 4. Coronary Angiogram in Right Anterior Oblique (RAO) Cranial view with collateral blood vessels from RCA to LAD as indicated by arrow high-intensity statin and unfractionated heparin and was admitted to coronary care unit for further management and observation.

\section{Discussion}

Myocardial infarction due to Blunt Chest Trauma (BCT) is rate but life-threatening condition. Because it has a low incidence, high suspicion is essential in patients with chest pain presenting after BCT. Most of the patients have EKG abnormalities which are nonspecific, but STEMI pattern can be seen in up to $2 \%$ of them [1]. Elevated cardiac markers including troponin and CK-MB and wall motion abnormalities seen on echocardiography do not help to differentiate between myocardial contusion and STEMI. The mechanisms of acute STEMI in the BCT setting include intimal tear, dissection, spasm, vessel rupture, plaque rupture, external compression from epicardial hematoma [2]. This can be explained by direct contusion and shearing forces during trauma. Majority of the cases reported are due to motor vehicle accidents, so this condition is seen more commonly in young men than women.

The affected arteries are Left anterior descending (LAD), right coronary artery (RCA) and circumflex $(\mathrm{Cx})$, in descending order [3]. There are no specific guidelines though in general therapeutic options include coronary angioplasty and stenting, by-pass surgery, thrombolytic and conservative treatment. In most of these reported cases were immediately evaluated by the medical team as a part of trauma protocol and got the necessary cardiac care intime. Coronary dissection remains the most common etiology for AMI post BCT. In our patient, because of the nature of the physical assault, clinical examination was misleading towards musculoskeletal chest pain/tenderness [4]. Elevated cardiac markers and Echo did not help to differentiate between MI and contusion. Persistent EKG changes was the clue towards rushing the patient to cath lab. According to the STEMI guidelines, PCI of a totally occluded infarct artery 24 hours after symptom onset in stable patients without signs of ischemia is class-III recommendation [5]. Therefore, our patient was not revascularized immediately but urgent cath was indicated to evaluate for any mechanical complication. There was no angiographic evidence of coronary dissection or atherosclerosis of remaining coronary arteries.

The etiology of acute thrombosis remains unclear through we can suspect underlying psychological stressors to contribute to the plague formation.

\section{Learning objectives}

Chest pain after BCT usually indicates musculoskeletal injury but cardiac damage should be kept in mind and be promptly evaluated by simple diagnostic tools like EKG and Echo. If the diagnosis of STEMI is made, coronary angiography should be performed as soon as possible, and management be given to a patient according to type of coronary artery lesion.

\section{References}

1. Berk WA (1987) ECG findings in non-penetrating chest trauma: a review. J Emerg Med 5: 209-215. [Crossref]

2. Christensen MD, Nielsen PE, Sleight P (2006) Prior blunt chest trauma may be a cause of single vessel coronary disease, hypothesis and review. Int J Cardiol 108: 1-5. [Crossref]

3. Oren A, Bar-Shlomo B, Stern S (1976) Acute coronary occlusion following blunt injury to the chest in the absence of coronary atherosclerosis. Am Heart J 92: 501-505. [Crossref] 
4. Abdolrahimi SA, Sanati HR, Ansari-Ramandi MM, Heris SO, Maadani M (2016) Acute Myocardial Infarction Following Blunt Chest Trauma and Coronary Artery Dissection. J Clin Diagn Res 10: OD14-OD15. [Crossref]
5. Lai CH, Ma T, Chang TC, Chang MUH, Chou P, et al. (2006) A case of blunt chest trauma induced acute myocardial infarction involving two vessels. Int Heart J 47: 639643. [Crossref]

Copyright: $(02021$ Jadhav P. This is an open-access article distributed under the terms of the Creative Commons Attribution License, which permits unrestricted use, distribution, and reproduction in any medium, provided the original author and source are credited. 